\title{
Diabetes and hypertension
}

\author{
Rodrigo M Lago, Premranjan P Singh and Richard W Nesto
}

The incidence and prevalence of type 2 diabetes are increasing; ${ }^{1,2}$ it is projected that the total number of people with diabetes will rise from 171 million in 2000 to 366 million by 2030. The number of adults with hypertension is predicted to increase by $60 \%$ to a total of 1.56 billion people by 2025 . Hypertension affects approximately $70 \%$ of patients with diabetes and is approximately twice as common in persons with diabetes as in those without. ${ }^{3}$ The prevalence of coexistent hypertension and diabetes varies across different ethnic, racial, and social groups. Importantly, hypertension in patients with diabetes causes a significant increase in the risk of vascular complications in this population, and together both conditions predispose to chronic kidney disease. ${ }^{4,5}$ The overlap between hypertension and diabetes substantially increases the risk of ischemic cerebrovascular disease, retinopathy, and sexual dysfunction. ${ }^{6}$ Diabetes mellitus is an independent risk factor for coronary artery disease, and the risk is markedly increased when hypertension is present.

Diabetic nephropathy is the commonest cause of hypertension in patients with type 1 diabetes. Patients with type 2 diabetes can develop renal disease, but hypertension commonly occurs without abnormal renal function and is often associated with central obesity. Insulin resistance and diabetes can precipitate hypertension by stimulating the sympathetic nervous system and the reninangiotensin system, and promoting sodium retention. Diabetes is also associated with increased proliferation of vascular smooth muscle cells. High blood glucose and elevated blood pressure can impair vascular endothelial cells, leading to increased oxidative stress. Patients with diabetes also have increased vascular reactivity.

Both hypertension and diabetes should be diagnosed early and treated aggressively to prevent associated microvascular and macrovascular morbidity and mortality. ${ }^{7-11}$ The
The

importance

of the topic

is such that a

free online Web

Collection on

the subject of

diabetes and

hypertension

has been

compiled... .

RM Lago and PP Singh are Fellows in the Department of Cardiovascular Medicine at Lahey Clinic Medical Center in Burlington, $M A$, USA. RW Nesto is an Advisory Board member of Nature Clinical Practice Endocrinology \& Metabolism.

\section{Competing interests} The authors declared an association with the following companies: GlaxoSmithKline, Takeda. See the article online for full details of the relationship.

www.nature.com/clinicalpractice doi:10.1038/ncpendmet0638
UK Prospective Diabetes Study showed that blood pressure control helps to avoid cardiovascular complications in patients with type 2 diabetes: ${ }^{12,13}$ each $10 \mathrm{mmHg}$ decrease in mean systolic blood pressure was associated with $12 \%$ reduction in the risk for any complication related to diabetes, $15 \%$ reduction in deaths related to diabetes, $11 \%$ reduction in myocardial infarction, and 13\% reduction in microvascular complications. The Heart Outcomes Prevention Evaluation study showed that inhibition of angiotensinconverting enzyme in patients with type 2 diabetes reduces the risk of vascular complications. ${ }^{14}$ Drug therapy is required in the management of these patients, but lifestyle modification and weight management are key components to reduce glycemia and control blood pressure.

There are particular subpopulations in which the coexistence of hypertension and diabetes can pose serious risks. Pregnant women with diabetes and hypertension are at risk for pre-eclampsia. Children with type 1 diabetes and hypertension are particularly vulnerable to end-organ disease. The increase in incidence of childhood type 2 diabetes is worrisome, as cardiovascular risk factors early in life can cause accelerated atherosclerosis with aging.

In addition to a major impact on clinical care, quality of life and public health, diabetes and hypertension account for significant health care expenditure. The compelling evidence linking these diseases and, mostly, its association and interaction with morbid conditions prompt clinical awareness. The importance of the topic is such that a free online Web Collection on the subject of diabetes and hypertension has been compiled, featuring articles published by the Nature Publishing Group. This and Supplementary information in the form of a list of references cited in this article are available on the Nature Clinical Practice Endocrinology \& Metabolism website. 